\title{
Double occupancy errors in quantum computing operations: Corrections to adiabaticity
}

\author{
Ryan Requist, ${ }^{1, *}$ John Schliemann, ${ }^{2, \dagger}$ Alexander G. Abanov, ${ }^{1, \$}$ and Daniel Loss ${ }^{2, \S}$ \\ ${ }^{1}$ Department of Physics and Astronomy, Stony Brook University, Stony Brook, New York 11794-3840, USA \\ ${ }^{2}$ Department of Physics and Astronomy, University of Basel, Klingelbergstrasse 82, CH-4056 Basel, Switzerland
}

(Received 14 September 2004; published 21 March 2005)

\begin{abstract}
We study the corrections to adiabatic dynamics of two coupled quantum dot spin qubits, each dot singly occupied with an electron, in the context of a quantum computing operation. Tunneling causes double occupancy at the conclusion of an operation and constitutes a processing error. We model the gate operation with an effective two-level system, where nonadiabatic transitions correspond to double occupancy. The model is integrable and possesses three independent parameters. We confirm the accuracy of Dykhne's formula, a nonperturbative estimate of transitions, and discuss physically intuitive conditions for its validity. Our semiclassical results are in excellent agreement with numerical simulations of the exact time evolution. A similar approach applies to two-level systems in different contexts.
\end{abstract}

DOI: 10.1103/PhysRevB.71.115315

PACS number(s): 03.67.Lx, 03.65.Sq, 73.23.Hk

\section{INTRODUCTION}

Quantum information processing is an active and fascinating direction of research with participation from various fields of physics and neighboring scientific disciplines. ${ }^{1}$ This extraordinary interest has generated a fairly vast amount of theoretical and experimental studies. Possible experimental realizations of quantum information processing are presently being investigated. Among the different approaches, those in a solid-state setting are attractive, because they offer the potential of scalability - the integration of a large number of quantum gates into a quantum computer once the individual gates and qubits are established. With that in mind, several proposals for using electron and/or nuclear spins in solidstate systems have been put forward in recent years. ${ }^{2-7}$ Specifically, in Ref. 2 it was proposed to use the spin of electrons residing in semiconductor quantum dots as qubits. ${ }^{8-15}$ In this paper we revisit the quantum dynamics of gate operations between qubits of this type. Such two-qubit operations are performed by varying the amplitude of electron tunneling between the dots via external electric potentials. In a generic scenario, the tunneling amplitude between the dots is zero (or, more precisely, exponentially small) before and after the gate operation, while it is finite and appreciable during such a process. Thus, the typical time dependence of the tunneling amplitude is a pulse roughly characterized by its duration, amplitude, and ramp time (see Fig. 1). During such a pulse, the tunneling amplitude is finite and essentially constant, and both electrons can explore the total system of two quantum dots. Therefore, their indistinguishable fermionic character is of relevance. ${ }^{10,16,17}$ In particular, in such gate operations entanglement-like quantum correlations arise which require a description different from the usual entanglement between distinguishable parties (Alice, Bob, ...) in bipartite (or multipartite) systems. In such a case the proper statistics of the indistinguishable particles has to be taken into account. ${ }^{10,16,17}$

Another important aspect of having a finite (as opposed to infinitely high) tunneling barrier between the dots is that it necessarily leads to (partially) doubly occupied states in the two-electron wave function, i.e., contributions to the wave function where both electrons are on the same dot (having different spins) occur with finite amplitude. Doubly occupied states which arise as the result of a measurement after the gate operation destroy the information in those qubits and lead to errors in the information processing. Therefore, it is desirable to reduce the probability of such errors, i.e., the occurrence of doubly occupied states, in the resulting twoelectron state after the gate operation, while it is necessarily finite during the operation. ${ }^{9,10}$ If the error probability can be sufficiently reduced, error events can be tolerable and handled with quantum error correction schemes. An effective way of guaranteeing error suppression is to maintain nearly adiabatic time evolution. Doubly occupied states then correspond to corrections to adiabatic evolution, which are often called "nonadiabatic transitions." Numerical simulations ${ }^{10}$ have shown that the adiabatic region, in terms of the pulse parameters such as ramp time and amplitude, is rather large. On a heuristic level, this numerical result is plausible on the basis of the classic papers on adiabatic quantum motion in two-level systems by Landau, ${ }^{18}$ Zener, ${ }^{19}$ Stueckelberg, ${ }^{20}$ and Rosen and Zener. ${ }^{21}$ For an overview see Ref. 22.

In this work we study the quantum dynamics of the twoqubit gate operations described above and use Dykhne's semiclassical result to estimate the probability of nonadiabatic transition. ${ }^{23}$ The applicability of Dykhne's formula is analyzed from the standpoint of the theory of semiclassical approximations. These semiclassical estimates are found to be in excellent agreement with numerical simulations of the exact time evolution. Moreover, in a certain limit our model is integrable, allowing us to explicitly calculate and interpret the corrections to Dykhne's formula.

This paper is organized as follows. Section II reviews the derivation ${ }^{10}$ of an effective two-level model. In Sec. III, we present our main result-the asymptotic estimate of double occupancy, which in Sec. IV is compared with an integrable model and a numerical integration of the Schrödinger equation. In the Appendix, we construct the scattering matrix for the integrable model, which has three independent parameters. 


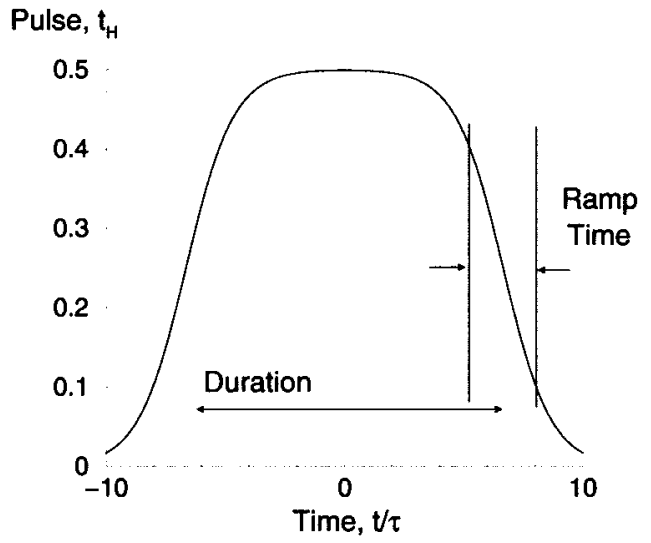

FIG. 1. A realistic profile of the tunneling pulse (23), labeled with the characteristic duration $(T \approx 13 \tau)$ and ramp time scales. The pulse shown has dimensionless strength $\delta=\frac{1}{2}$.

\section{MAPPING TO AN EFFECTIVE TWO-LEVEL SYSTEM}

For the purpose of studying double occupancy it is practical to examine the dynamics of the quantum gate operation in a subspace spanned by singly and doubly occupied states. Following Ref. 10 with only minor changes of notation, we now detail how to reduce the description of a system of two coupled quantum dot spin qubits to an effective two-level Hamiltonian. The system is described by a Hamiltonian of the form $\mathcal{H}=T+C$, where $C$ denotes the Coulomb repulsion between the electrons and $T=\sum_{i=1,2} h_{i}$ is the single-particle part with

$$
h_{i}=\frac{1}{2 m}\left(\vec{p}_{i}+\frac{e}{c} \vec{A}\left(\vec{r}_{i}\right)\right)^{2}+V\left(\vec{r}_{i}\right) .
$$

The single-particle Hamiltonian $h_{i}$ describes electron dynamics confined to the $x y$ plane in a perpendicular magnetic field $\vec{B}$. The effective mass $m$ is a material dependent parameter. The coupling of the dots (which includes tunneling) is modeled by a quartic potential

$$
V(\vec{r})=V(x, y)=\frac{m \omega_{0}^{2}}{2}\left(\frac{1}{4 a^{2}}\left(x^{2}-a^{2}\right)^{2}+y^{2}\right),
$$

which separates into two harmonic wells of frequency $\omega_{0}$ (one for each dot) in the limit $a \gg a_{0}$, where $a$ is half the distance between the dots and $a_{0}=\sqrt{\hbar / m \omega_{0}}$ is the effective Bohr radius of a dot.

Following Burkard et al. ${ }^{8}$ we employ the Hund-Mulliken method of molecular orbits to describe the low-lying spectrum of our system. This approach concentrates on the lowest orbital states in each dot and is an extension of the HeitlerLondon method. ${ }^{8}$ [In the following, we assume for simplicity that $\hbar \omega \gg U_{H}$ i.e., (single particle orbital level spacing) $\gg$ (quantum dot charging energy), so that orbital excitations can be safely neglected. Such a situation is reached for sufficiently small quantum dots. $\left.{ }^{12}\right]$ The Hund-Mulliken approach accounts for the fact that both electrons can, in the presence of a finite tunneling amplitude, explore the entire system of the two dots, and therefore adequately includes the possibility of doubly occupied states. In the usual symmetric gauge
$\vec{A}=B(-y, x, 0) / 2$ the Fock-Darwin ground state of a single dot with harmonic confinement centered around $\vec{r}$ $=( \pm a, 0,0)$ reads

$$
\begin{aligned}
\varphi_{ \pm a}(x, y)= & \sqrt{\frac{m \omega}{\pi \hbar}} \exp \left(-\frac{m \omega}{2 \hbar}\left[(x \mp a)^{2}+y^{2}\right]\right) \\
& \times \exp \left(\mp \frac{i}{2} y \frac{a}{l_{B}^{2}}\right),
\end{aligned}
$$

where $l_{B}=\sqrt{\hbar c / e B}$ is the magnetic length, and the frequency $\omega$ is given by $\omega^{2}=\omega_{0}^{2}+\left(\omega_{L} / 2\right)^{2}$ where $\omega_{L}=e B / m c$ is the usual Larmor frequency. From these nonorthogonal singleparticle states we construct the orthonormalized states $|A\rangle$ and $|B\rangle$ with wave functions

$$
\begin{aligned}
& \langle\vec{r} \mid A\rangle=\frac{1}{\sqrt{1-2 S g+g^{2}}}\left(\varphi_{+a}-g \varphi_{-a}\right), \\
& \langle\vec{r} \mid B\rangle=\frac{1}{\sqrt{1-2 S g+g^{2}}}\left(\varphi_{-a}-g \varphi_{+a}\right),
\end{aligned}
$$

with $S$ being the overlap between the states (3) and $g=(1$ $\left.-\sqrt{1-S^{2}}\right) / S$. For appropriate values of system parameters such as the interdot distance and the external magnetic field, the overlap $S$ becomes exponentially small. ${ }^{8}$ In this limit an electron in one of the states $|A\rangle,|B\rangle$ is predominantly localized around $\vec{r}=( \pm a, 0,0)$. In the following we consider this case and use these states as basis states to define qubits, i.e., qubits are realized by the spin state of an electron in either orbital $|A\rangle$ or orbital $|B\rangle$.

An appropriate basis set for the six-dimensional twoparticle Hilbert space is given (using standard notation) by the three spin singlets,

$$
\begin{aligned}
& \left|S_{1}\right\rangle=\frac{1}{\sqrt{2}}\left(c_{A \uparrow}^{+} c_{B \downarrow}^{+}-c_{A \downarrow}^{+} c_{B \uparrow}^{+}\right)|0\rangle, \\
& \left|S_{2}\right\rangle=\frac{1}{\sqrt{2}}\left(c_{A \uparrow}^{+} c_{A \downarrow}^{+}+c_{B \uparrow}^{+} c_{B \downarrow}^{+}\right)|0\rangle, \\
& \left|S_{3}\right\rangle=\frac{1}{\sqrt{2}}\left(c_{A \uparrow}^{+} c_{A \downarrow}^{+}-c_{B \uparrow}^{+} c_{B \downarrow}^{+}\right)|0\rangle,
\end{aligned}
$$

and the triplet multiplet,

$$
\begin{gathered}
\left|T^{-1}\right\rangle=c_{A \downarrow}^{+} c_{B \downarrow}^{+}|0\rangle, \\
\left|T^{0}\right\rangle=\frac{1}{\sqrt{2}}\left(c_{A \uparrow}^{+} c_{B \downarrow}^{+}+c_{A \downarrow}^{+} c_{B \uparrow}^{+}\right)|0\rangle, \\
\left|T^{1}\right\rangle=c_{A \uparrow}^{+} c_{B \uparrow}^{+}|0\rangle .
\end{gathered}
$$

As the Hamiltonian conserves spin, the three triplet states are degenerate eigenstates (typically we can ignore possible Zeeman splittings ${ }^{8}$ ) and have the eigenvalue

$$
\varepsilon_{\text {trip }}=2 \varepsilon_{1}+V_{-},
$$

where we have defined 


$$
\varepsilon_{1}=\left\langle A\left|h_{1}\right| A\right\rangle=\left\langle B\left|h_{1}\right| B\right\rangle
$$

and the expectation value of Coulomb energy,

$$
V_{-}=\left\langle T^{\alpha}|C| T^{\alpha}\right\rangle, \quad V_{+}=\left\langle S_{1}|C| S_{1}\right\rangle .
$$

An important further observation is that, as a consequence of inversion symmetry along the axis connecting the dots, the Hamiltonian does not have any nonzero matrix elements between the singlet state $\left|S_{3}\right\rangle$ and other states. Hence, $\left|S_{3}\right\rangle$ is, independently of the system parameters, an eigenstate. The eigenvalues of the triplet and $\left|S_{3}\right\rangle$, however, do depend on system parameters. The Hamiltonian acting on the remaining space spanned by $\left|S_{1}\right\rangle$ and $\left|S_{2}\right\rangle$ can be written as

$$
\mathcal{H}=2 \varepsilon_{1}+\frac{1}{2} U_{H}+V_{+}-\frac{U_{H}}{2}\left(\begin{array}{cc}
1 & t_{H} \\
t_{H} & -1
\end{array}\right),
$$

where

$$
t_{H}=-\frac{4}{U_{H}}\left(\left\langle A\left|h_{1}\right| B\right\rangle+\frac{1}{2}\left\langle S_{2}|C| S_{1}\right\rangle\right)
$$

and

$$
U_{H}=\left\langle S_{2}|C| S_{2}\right\rangle-V_{+}
$$

The nontrivial part of Eq. (15) is a simple Hubbard Hamiltonian on two sites and can be identified as the Hamiltonian of a pseudospin- $\frac{1}{2}$ object in a pseudomagnetic field having a component $U_{H}$ in the $\hat{z}$ direction and $U_{H} t_{H}$ in the $\hat{x}$ direction of pseudospin space. (Note that this pseudospin is not related to the spin degree of freedom which constitutes the qubit.) The basis states themselves are eigenstates only in the case of vanishing tunneling amplitude $t_{H}$ where $\left|S_{1}\right\rangle$ is the ground state and $\left|S_{2}\right\rangle$ is a higher lying state due of the Coulomb (Hubbard) energy. In all other cases, the ground state has an admixture of doubly occupied states contained in $\left|S_{2}\right\rangle$. The energy gap between the triplet and the singlet ground state is

$$
\varepsilon_{\text {trip }}-\varepsilon_{\text {gs }}=V_{-}-V_{+}-\frac{U_{H}}{2}+\frac{U_{H}}{2} \sqrt{1+t_{H}^{2}} .
$$

A key challenge for state-of-the-art quantum information processing is the construction of systems composed of two coupled quantum dots which can be coupled to perform swap operations $\mathcal{U}_{S W}$, i.e., unitary two-qubit operations which interchange the spin states (qubits) of the electrons on the two dots. By combining the "square root" $\mathcal{U}_{S W}^{1 / 2}$ of such a swap with other isolated-qubit manipulations one can construct a quantum XOR gate. A quantum XOR gate, along with isolatedqubit operations, has been shown to be sufficient for the implementation of any quantum algorithm. ${ }^{24}$ Hence a practical and reliable realization of a swap gate would be an important step toward the fabrication of a solid-state quantum computer. A swap operation in the present system is a unitary transformation which turns a state having the qubits in different states, say,

$$
c_{A \uparrow}^{+} c_{B \downarrow}^{+}|0\rangle=\frac{1}{\sqrt{2}}\left(\left|T^{0}\right\rangle+\left|S_{1}\right\rangle\right),
$$

into a state where the contents of the qubits are interchanged,

$$
c_{A \downarrow}^{+} c_{B \uparrow}^{+}|0\rangle=\frac{1}{\sqrt{2}}\left(\left|T^{0}\right\rangle-\left|S_{1}\right\rangle\right) .
$$

These two states are eigenstates in the case $V_{+}=V_{-}$and $t_{H}$ $=0$ for which the singlet-triplet splitting vanishes.

As discussed in Refs. 2, 8, and 10, swapping may be achieved by the action of a gate that lowers the potential barrier between the quantum dots. If the duration and amplitude of a tunneling pulse are adjusted appropriately, the relative dynamical phase between the singlet and the triplet states accumulates a shift of $\pi$,

$$
\frac{1}{\hbar} \int_{-\infty}^{\infty} d t\left[\varepsilon_{\text {trip }}(t)-\varepsilon_{\mathrm{gs}}(t)\right]=\pi
$$

and the swapping operation between states (19) and (20) is performed. However, during the operation the state $\left|S_{1}\right\rangle$ is coupled to $\left|S_{2}\right\rangle$, and they evolve according to Eq. (15). Double occupancy errors are thus generically introduced.

The reduction of the dynamics to the time evolution of a two-level system relies on the fact that the system has inversion symmetry along the $\hat{x}$ axis in real space connecting the dots. This symmetry can be broken if odd powers of the particle coordinates $x_{i}$ are added to the Hamiltonian (1), for example, the potential of a homogeneous electric field. The breaking of inversion symmetry introduces additional matrix elements between $\left|S_{3}\right\rangle$ and the other two singlets leading to an effective three-level Hamiltonian. However, as it was shown in Ref. 10, this more inclusive Hamiltonian has qualitatively the same properties concerning nonadiabatic dynamics as the two-level system on which we shall concentrate in the following.

So far we have not considered a possible Zeeman coupling to the electron spin. This would not change the situation essentially since all states involved in the swapping process $\left(\left|T^{0}\right\rangle,\left|S_{1}\right\rangle,\left|S_{2}\right\rangle\right.$, and possibly $\left.\left|S_{3}\right\rangle\right)$ have the total spin quantum number $S^{z}=0$.

\section{ANALYSIS OF NONADIABATIC TRANSITIONS}

In this section we use Dykhne's formula for nonadiabatic transitions to derive an asymptotic expression for the probability of final double occupancy, given physically motivated properties of the two-qubit operation.

As described in the preceding section, the modulation of the tunneling barrier during the swapping process induces a coupling between the singly occupied qubit state $\left|S_{1}\right\rangle$ and the doubly occupied state $\left|S_{2}\right\rangle$. Their dynamics are governed by the effective Hamiltonian

$$
\mathcal{H}_{\text {eff }}=-\frac{U_{H}}{2}\left(\begin{array}{cc}
1 & t_{H} \\
t_{H} & -1
\end{array}\right)
$$

in the $\left|S_{1,2}\right\rangle$ basis. The terms omitted from Eq. (15) do not contribute to transitions, because the identity operator in the $\left|S_{1,2}\right\rangle$ basis commutes with the remainder of the Hamiltonian. The large energy offset $U_{H}$ between singly and doubly occupied states, primarily due to the Coulomb repulsion, is perturbed only by an exponentially small additive quantity (proportional to the overlap, $S$ ) during the swapping operation 


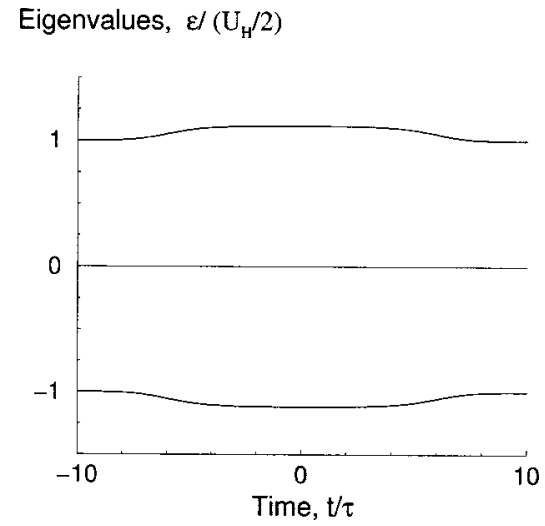

FIG. 2. A profile of the instantaneous eigenvalues $\pm \varepsilon(t)$ corresponding to $\lambda=2$ and the pulse shown in Fig. 1 .

and is hereafter assumed to be a constant. Our specification of the pulse (Fig. 1)

$$
t_{H}(t)=\frac{\delta}{1+\frac{\cosh (t / \tau)}{\cosh (T / 2 \tau)}}
$$

with dimensionless strength $\delta$ is considered to realistically reflect the tunneling amplitude that would arise from a modulation of the gate potential. ${ }^{10}$ The exponential dependence of the ramping near $t= \pm T / 2$ has its origin in the exponential sensitivity of the coupling to the gate voltage and in turn the exponential decay of the single-particle wave functions (3) in the interdot region. ${ }^{25}$ The pulse mimics a step of duration $T$ and magnitude $\delta U_{H} / 2$, whose ramping on and off has a characteristic time $\tau$. The perturbation of the instantaneous eigenvalues by the pulse is shown in Fig. 2 .

The Schrödinger equation is

$$
i \hbar \frac{d}{d t}|\psi(t)\rangle=\mathcal{H}_{\mathrm{eff}}(t)|\psi(t)\rangle .
$$

Our task is to find the component of double occupancy in the final state, $\left\langle S_{2} \mid \psi(\infty)\right\rangle$, given that the prepared state is purely singly occupied, $\left|\left\langle S_{1} \mid \psi(-\infty)\right\rangle\right|=1$.

Our model involves three dimensionless scales, assigned for our purposes as follows: $\delta, \lambda \equiv U_{H} \tau / 2 \hbar$, and $\eta=T / \tau$. Presently, the case of interest is

$$
\lambda \gg 1, \quad \eta \gg 1 .
$$

The first of these conditions reflects the adiabaticity of the problem. The second requires that the ramping on and ramping off of the pulse be temporally well-separated and distinct events.

Let us pause and for this paragraph review the familiar notions of transitions under the action of a time-dependent perturbation. The pulse acts as a transient perturbation and otherwise the Hamiltonian (22) is diagonal. By force of the adiabatic theorem, the probability of transition among eigenstates vanishes in the limit $\tau \rightarrow \infty$, where the ramping on and off of the pulse is adiabatic. In the zeroth order of adiabatic perturbation theory, there are no transitions, and the leading behavior of the general solution is simply the dynamical phase of each component eigenstate

$$
\begin{aligned}
|\psi(t)\rangle \approx & \exp \left[\frac{i}{\hbar} \int_{-\infty}^{t} d t^{\prime} \varepsilon\left(t^{\prime}\right)\right]\left|\xi_{1}(t)\right\rangle\left\langle\xi_{1}(-\infty) \mid \psi(-\infty)\right\rangle \\
& +\exp \left[-\frac{i}{\hbar} \int_{-\infty}^{t} d t^{\prime} \varepsilon\left(t^{\prime}\right)\right]\left|\xi_{2}(t)\right\rangle\left\langle\xi_{2}(-\infty) \mid \psi(-\infty)\right\rangle,
\end{aligned}
$$

where $\left|\xi_{1,2}(t)\right\rangle$ are the instantaneous eigenstates [given explicitly in Eq. (54)] of Hamiltonian (22) corresponding to eigenvalues

$$
\mp \varepsilon(t)=\mp \frac{U_{H}}{2} \sqrt{1+t_{H}^{2}}
$$

respectively. In general, the approximate solution could also include a factor representing Berry phase. However, for a real symmetric Hamiltonian such as Eq. (22), Berry phase is irrelevant, because the Hamiltonian has an inherent planarity. In pseudospin one-half notation, $\mathcal{H}_{\text {eff }}=\vec{H}(t) \cdot \vec{\sigma}$, the time evolution of the pseudomagnetic field $\vec{H}(t)$ is in a plane. If the azimuthal axis (north pole) is chosen to lie within that plane, the solid angle subtended by the pseudomagnetic field vanishes identically. Although Berry phase is out of consideration, there are interesting circumstances where Berry phase is relevant to transitions. It can correct the transition amplitude $^{26}$ and produce topological selection rules for spin tunneling. ${ }^{27,28}$ Our problem is one of a class initiated by the work of Landau, Zener, and Stueckelberg. ${ }^{18-20}$ However, we emphasize that for our model [with the pulse specified as Eq. (23)] the linearization of Hamiltonian matrix elements near the times where adiabaticity is most severely violated is not applicable and leads to an incorrect result. As we will see the shape of the pulse is important.

\section{A. Application of Dykhne's formula}

Returning to our model, we observe that if the time interval $t \in(-\infty, \infty)$ is divided into two domains $t<0$ and $t>0$, and in the limit $\eta \equiv T / \tau \gg 1$, the pulse (23) is approximated by

$$
t_{H}(t) \approx \begin{cases}\frac{\delta}{1+e^{-(t / \tau)-(T / 2 \tau)},} & t<0 \\ \frac{\delta}{1+e^{(t / \tau)-(T / 2 \tau)}}, & t>0 .\end{cases}
$$

In each domain the pulse behaves as a step, and the dynamics are integrable (see Sec. IV). We will focus first on the interval $t<0$, where the probability of transition to a doubly occupied state $P_{<}$may be estimated with Dykhne's formula ${ }^{23}$ 


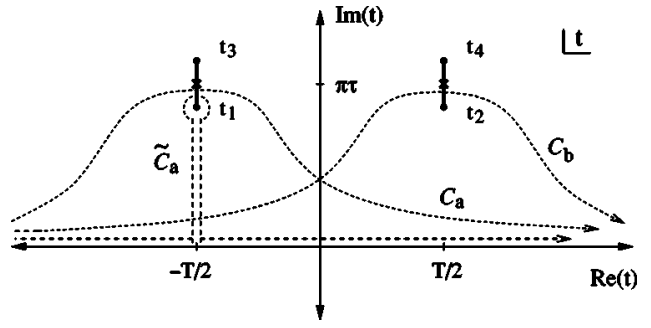

FIG. 3. The analytic structure of the function $\varepsilon(t)$ shown only in a segment of the upper half plane. The contour $\mathcal{C}_{a}$ is associated with transitions that occur due to the ramping on of the pulse, while contour $\mathcal{C}_{b}$ is associated with the ramping off. By Cauchy's theorem, an integral on the contour $\mathcal{C}_{a}$ is equal to the integral on the contour $\widetilde{\mathcal{C}}_{a}$. Bold lines represent branch cuts, dots represent branch points, and poles are denoted with $\mathrm{a} \times$.

$$
P_{<}=\left|\left\langle S_{2} \mid \psi(0)\right\rangle\right|^{2} \sim \exp \left[-\frac{4}{\hbar} \operatorname{Im} \int_{\operatorname{Re}\left(t_{1}\right)}^{t_{1}} d z \varepsilon(z)\right],
$$

where the approximation (28) is used implicitly for the instantaneous eigenenergies $\mp \varepsilon(t)$ defined above by Eq. (27). The turning point $t=t_{1}$, given explicitly below, is a complex root of the function $\varepsilon(t)$; in other words, it is an intersection of the energy surfaces of the two instantaneous ("frozen") eigenstates. Our model is the patching together of two domains of time, and transitions that occur during $t<0$ and $t$ $>0$ interfere. The expression for the probability of transition during the time evolution from $t=-\infty$ to $t=\infty$ is

$$
\begin{aligned}
& P=\left|\left\langle S_{2} \mid \psi(\infty)\right\rangle\right|^{2} \\
& \sim\left|\exp \left[\frac{i}{\hbar} \int_{C_{a}} d z \varepsilon(z)\right]+\exp \left[\frac{i}{\hbar} \int_{C_{b}} d z \varepsilon(z)\right]\right|^{2} \\
& \sim \mid \exp \left[\frac{i}{\hbar} \operatorname{Re} \int_{C_{a}} d z \varepsilon(z)\right] \\
& \quad \times \exp \left[-\frac{2}{\hbar} \operatorname{Im} \int_{\operatorname{Re}\left(t_{1}\right)}^{t_{1}} d z \varepsilon(z)\right]+\exp \left[\frac{i}{\hbar} \operatorname{Re} \int_{C_{b}} d z \varepsilon(z)\right] \\
& \quad \times\left.\exp \left[-\frac{2}{\hbar} \operatorname{Im} \int_{\operatorname{Re}\left(t_{2}\right)}^{t_{2}} d z \varepsilon(z)\right]\right|^{2} \\
&=4 \sin ^{2}\left(\frac{1}{\hbar} \operatorname{Re} \int_{t_{1}}^{t_{2}} d z \varepsilon(z)\right) P_{<},
\end{aligned}
$$

where the contours $\mathcal{C}_{a, b}$ are shown in Fig. 3, and according to the sign of the integration variable, $\operatorname{sgn}(\operatorname{Re} z)$, one or the other of the approximations (28) is used. The turning points $t=t_{1,2}$ appearing in the limits of integration of Eq. (30) are chosen as the two roots of $\varepsilon(t)$ that are closest to and above the real time axis (see Fig. 3),

$$
t_{1,2}=\mp\left(\frac{T}{2}+\tau \ln \left(\sqrt{1+\delta^{2}}\right)\right)+i \tau[\pi-\arctan (\delta)] .
$$

They are nonreal because the Hamiltonian (22) is nondegenerate for real times. Equation (31) follows from Eq. (30), because the symmetry of the pulse implies $\operatorname{Im}\left(t_{1}\right)=\operatorname{Im}\left(t_{2}\right)$

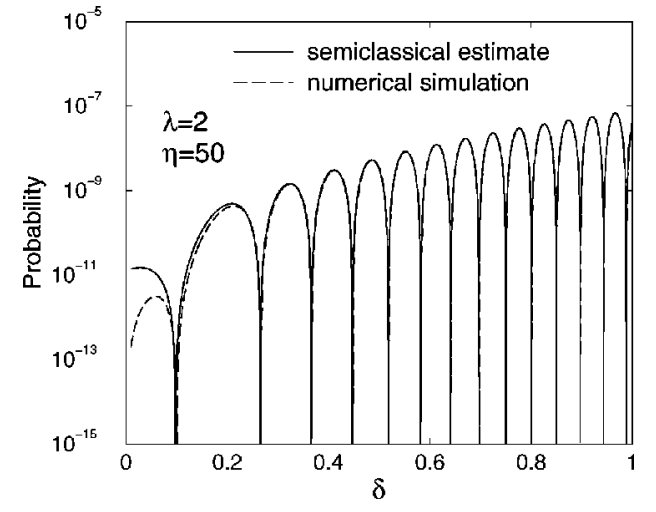

FIG. 4. The probability for nonadiabatic transitions for $\lambda=2$ and $\eta=50$ as a function of $\delta$. We compare our semiclassical estimate according to expression (35) with results from numerical simulations of the exact quantum mechanical time evolution as done in Ref. 35. The results are in excellent agreement.

and $P_{<}=P_{>}$. The oscillatory first factor of Eq. (31) is the interference of the dynamical phase of each term of Eq. (30). The magnitude of $P$ is dominated by the second factor $P<$ whose exponent is given by the following integral:

$$
\begin{aligned}
&-4 \lambda \operatorname{Im} \int_{\ln \left(\sqrt{1+\delta^{2}}\right)}^{\ln \left(\sqrt{1+\delta^{2}}\right)+i \pi-i \arctan (\delta)} d z\left[1+\left(\frac{\delta}{1+e^{z}}\right)^{2}\right]^{1 / 2} \\
&=-2 \pi \lambda\left(1+\sqrt{1+\delta^{2}}-\delta\right) .
\end{aligned}
$$

Substituting this result in Eq. (29) we have

$$
P_{<} \sim e^{-2 \pi \lambda\left(1+\sqrt{1+\delta^{2}}-\delta\right)} .
$$

From Eq. (31), we have our main result, an asymptotic estimate for the probability of final double occupancy,

$$
P \sim 4 \sin ^{2}\left(\frac{1}{\hbar} \operatorname{Re} \int_{t_{1}}^{t_{2}} d z \varepsilon(z)\right) e^{-2 \pi \lambda\left(1+\sqrt{1+\delta^{2}}-\delta\right)},
$$

which is shown as a function of $\delta$ in Fig. 4. The probability $P$ is characteristically nonperturbative in the adiabatic limit $\tau \rightarrow \infty$ with $U_{H}$ fixed, or equivalently $\lambda \rightarrow \infty$. Hence, the dimensionless quantity associated with the exponential suppression is $\lambda$ and has been called the "adiabaticity parameter." For $\eta \equiv T / \tau \gg 1$, the approximation (28) allows us to estimate the argument of the prefactor of Eq. (35) to exponential accuracy,

$$
\begin{aligned}
\frac{1}{\hbar} \operatorname{Re} \int_{t_{1}}^{t_{2}} d z \varepsilon(z)= & \sqrt{1+\delta^{2}} \lambda \eta-2 \lambda\left\{\ln \left(\sqrt{1+\delta^{2}}+1\right)\right. \\
& -\sqrt{1+\delta^{2}} \ln \left[2\left(1+\delta^{2}\right)\right]+\delta \ln \left(\sqrt{1+\delta^{2}}+\delta\right) \\
& \left.+\left(\sqrt{1+\delta^{2}}-1\right) \ln (\delta)\right\}+O\left(e^{-\eta / 2}\right)
\end{aligned}
$$

The oscillation with respect to the duration of the pulse $T$ is reminiscent of a similar factor in the Rosen-Zener model. The phenomenon of pulsed perturbations that return the full amplitude/occupation to the initial state has been studied in the context of atom-laser interactions. ${ }^{29-32}$ In Figs. 4-6, we compare our semiclassical estimate (35) with results from numerical simulations of the exact quantum mechanical time 


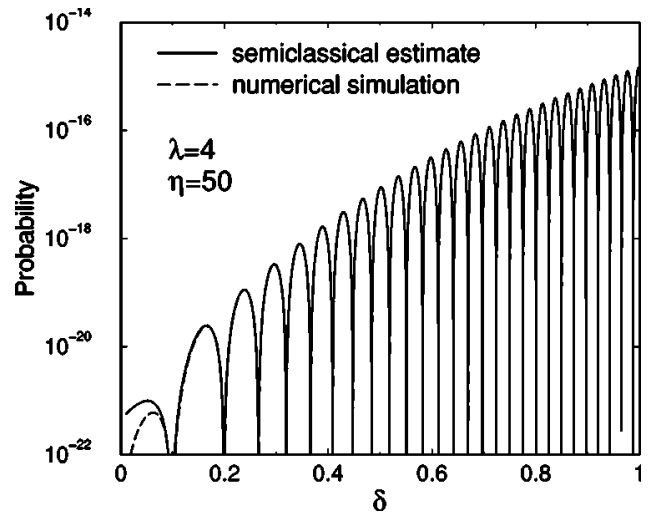

FIG. 5. The probability for nonadiabatic transitions for $\lambda=4$ and $\eta=50$ as a function of $\delta$.

evolution, following Ref. 10. Both results are in excellent agreement and differ only at very small $\delta$, i.e., for weak pulses. Of course, the nonadiabatic transition probability vanishes in this limit, whereas the semiclassical approximation breaks down (see Sec. III C). This regime is beyond the exponential accuracy of Dykhne's formula. The integrability of our model allows us to make precise statements about the form and magnitude of the corrections to Dykhne's formula (see Sec. IV). For example, in the limit $\lambda \gg 1$ and $\delta \lambda \ll 1$ we have from the expansion (63) that $P_{<} \sim(2 \pi \delta \lambda)^{2} e^{-4 \pi \lambda}$, while in the same limit the result of Dykhne's formula (35) gives only the exponential factor $e^{-4 \pi \lambda}$ without information about the prefactor. This explains a trend among Figs. 4-6, namely, the increasing range, in terms of $\delta$, of validity of Dykhne's formula with increasing $\lambda$. The value for the adiabaticity parameter $\lambda=2$, represented in Fig. 4, corresponds to a ramp time $\tau=4 \hbar / U_{H}$, which was identified in Ref. 10 as a practical lower bound to ensure sufficient adiabatic behavior in a gate operation between two quantum dot spin qubits. It is interesting that Dykhne's formula remains accurate for smaller values of $\lambda$ in particular $\lambda=1$ as seen in Fig. 6 . The reason is that the results (35) and (61) have an incidental factor of $2 \pi$ in the exponent, giving in practical terms the requirement for exponential suppression $2 \pi \lambda \gg 1$.

The expressions (34) and (35), along with Figs. 4-6, comprise our main results. For the remainder of this section, we will address the justification and limitations of these results.

\section{B. Origin of Dykhne's formula}

Dykhne derived a concise expression for nonadiabatic transitions from a local analysis of the Schrödinger equation in the vicinity of the turning point. ${ }^{23}$ Dykhne's formula can be viewed as a semiclassical approximation, and an elegant interpretation and proof was given by Hwang and Pechukas ${ }^{33}$ (see also Ref. 34). We will briefly discuss the key elements and scope of the proof. Their method was to study the solution of the Schrödinger equation in the complex plane of the independent variable, time. According to the adiabatic theorem, the projection of the solution onto any eigenstate other than the initial eigenstate approaches zero in the adiabatic limit. One might suppose that weak statement is all the adiabatic theorem can tell us about transitions; however, it does

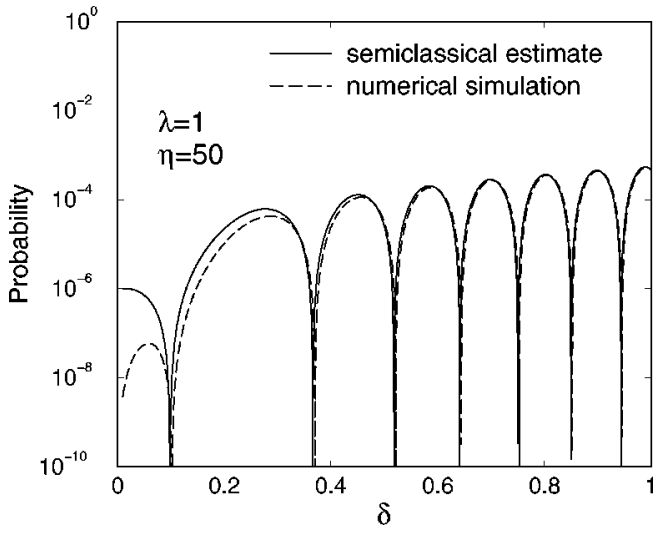

FIG. 6. The probability for nonadiabatic transitions for $\lambda=1$ and $\eta=50$ as a function of $\delta$.

not exhaust its capacities. The reason lies in the following: a basis of eigenstates $\left|\xi_{1,2}(t)\right\rangle$, when extended into the complex time plane, is multivalued. In particular, as a basis state is analytically continued across a branch cut of the function $\varepsilon(t)$, its long-time asymptotics are discontinuously changed. In accord with our above two-level problem, we uniquely specify the basis by its asymptotics,

$$
\left|\xi_{1,2}(t)\right\rangle \rightarrow\left|S_{1,2}\right\rangle \quad \text { as } \quad t \rightarrow \pm \infty
$$

The multivalued nature is not manifest on the real time axis, because owing to the nondegeneracy of the spectrum $\mp \varepsilon(t)$, the branch points are nonreal. We can choose a single-valued basis $\left|\widetilde{\xi}_{1,2}(t)\right\rangle$, which makes reference to $\left|\xi_{1,2}(t)\right\rangle$ but has fixed asymptotics, by defining rules for continuing the basis states across branch cuts. Equivalently, this new basis is said to be defined over a Riemann surface with sheets (copies of the complex time plane) corresponding to each of the two branches of the function $\left[\varepsilon(t)^{2}\right]^{1 / 2}$. (The Riemann surface $\mathcal{R}_{1}$ for the eigenstate basis $\left|\widetilde{\xi}_{1,2}(t)\right\rangle$ has four sheets, while the Riemann surface $\mathcal{R}_{2}$ for the function $\left[\varepsilon(t)^{2}\right]^{1 / 2}$ has two sheets. Of the four sheets of $\mathcal{R}_{1}$, two correspond to one branch of $\left[\varepsilon(t)^{2}\right]^{1 / 2}$ and the other two correspond to the other branch of $\left[\varepsilon(t)^{2}\right]^{1 / 2}$. Therefore, the phases $\alpha_{1,2}$ and $\beta_{1,2}$ of Eq. (38), though constant on each sheet, can assume different values on different sheets of $\mathcal{R}_{1}$.) Crossing a branch cut means passing to the other sheet of the Riemann surface. We assign the following relations among the eigenstates:

$$
\begin{gathered}
\left|\widetilde{\xi}_{1,2}(t)\right\rangle=e^{i \alpha_{1,2}}\left|\xi_{1,2}(t)\right\rangle, \quad t \in \text { sheet } 1 \text { of }\left[\varepsilon(t)^{2}\right]^{1 / 2}, \\
\left|\widetilde{\xi}_{1,2}(t)\right\rangle=e^{i \beta_{1,2}}\left|\xi_{2,1}(t)\right\rangle, \quad t \in \text { sheet } 2 \text { of }\left[\varepsilon(t)^{2}\right]^{1 / 2},
\end{gathered}
$$

where $\alpha_{1,2}$ and $\beta_{1,2}$ are phase definitions that are chosen to maintain continuity of the basis $\left|\widetilde{\xi}_{1,2}\right\rangle$ across the branch cut. Given $\left|\left\langle\widetilde{\xi}_{1}(-\infty) \mid \psi(-\infty)\right\rangle\right|=1$, the conclusion of the adiabatic theorem may be restated on a Riemann surface as

$$
\left|\left\langle\tilde{\xi}_{1}(t) \mid \psi(t)\right\rangle\right| \rightarrow 1 \quad \forall t \quad \text { as } \quad \tau \rightarrow \infty,
$$

where $\tau$ is the characteristic time scale for variation of $\mathcal{H}_{\text {eff }}(t)$. The only exception to Eq. (39) is for times within 


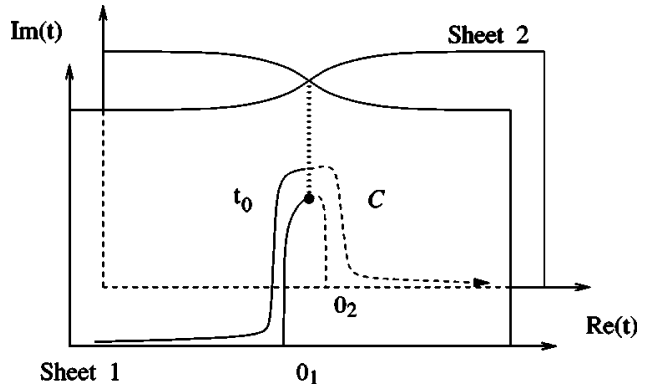

FIG. 7. An example of a Riemann surface with two sheets, a branch point at $t=t_{0}$ and a contour $\mathcal{C}$ corresponding to a transition.

$O\left(\tau \lambda^{-2 / 3}\right)$ of a turning point, for there the semiclassical criterion (45) is invalid. As remarked above, the zeroth-order approximation (26) of the solution as $\tau \rightarrow \infty$ is the dynamical phase. The zeroth-order approximation may be extended into the complex plane by evaluating the dynamical phase on a contour $\mathcal{C}$. Continuing with the above example, a state that is purely singly occupied at $t=-\infty$ is for complex time given by

$$
|\psi(t)\rangle \approx \exp \left\{-\frac{i}{\hbar} \int_{\mathcal{C}} d z[-\varepsilon(z)]\right\}\left|\tilde{\xi}_{1}(t)\right\rangle,
$$

where $\mathcal{C}$ is a contour from $z=-\infty$ to $z=t$. The amplitude of transition is readily obtained as the projection of the solution onto the doubly occupied state $\left|S_{2}\right\rangle$, as $t \rightarrow \infty$ on the second Riemann sheet (see Fig. 7) of $\left[\varepsilon(t)^{2}\right]^{1 / 2}$, i.e.,

$$
\begin{aligned}
\left\langle S_{2} \mid \psi(\infty)\right\rangle & =e^{-i \alpha_{1}}\left\langle\tilde{\xi}_{1}(\infty) \mid \psi(\infty)\right\rangle \\
& \approx e^{-i \alpha_{1}} \exp \left\{-\frac{i}{\hbar} \int_{\mathcal{C}} d z[-\varepsilon(z)]\right\},
\end{aligned}
$$

where the contour $\mathcal{C}$ crosses the branch cut emanating from the branch point that is closest to the real axis. Dykhne's formula is simply the square modulus of this amplitude.

In the adiabatic regime, in contrast to the perturbative regime, the leading contribution to transitions comes from the zeroth-order term of perturbation theory instead of the first-order term. By retaining only the zeroth-order term, it appears that we have neglected completely the coupling $t_{H}$ between states. However, the coupling enters implicitly in the multivalued function $\left[\varepsilon(t)^{2}\right]^{1 / 2}$ and influences the location of the turning points-the complex roots of $\varepsilon(t)$. Transition amplitudes are obtained by carefully considering the different branches of this function. In the following section, we consider the validity of keeping only the zeroth-order term.

\section{Validity and accuracy of Dykhne's formula}

The theory of semiclassical approximations, especially WKB analysis, provides a foundation from which to evaluate the validity of Dykhne's formula. The calculation of nonadiabatic transitions is closely related to the semiclassical approximation ${ }^{33}$ because the semiclassical limit $\hbar \rightarrow 0$ can be mathematically equivalent to the adiabatic limit $\tau \rightarrow \infty$. An essential element of the proof by Hwang and Pechukas is the existence of a complex time contour that (1) connects the two sheets of the Riemann surface and (2) on which the zeroth-order approximation of adiabatic perturbation theory is the correct leading behavior of the solution in the adiabatic limit. These are sufficient conditions for Dykhne's formula to give the correct asymptotic form of the transition probability in the adiabatic limit $\lambda \rightarrow \infty$. Having established the existence of such a contour, one can calculate a more precise value for the prefactor of Dykhne's formula by applying timedependent perturbation theory along the contour. We expect Dykhne's formula to break down when the contour ceases to exist. At the limit of its range of validity, the higher-order terms become comparable to the zeroth- order term. Introducing the unitary transformation $U$ that diagonalizes the Hamiltonian, i.e., $U^{\dagger} H U=\varepsilon \sigma_{3}$, we can write the Schrödinger equation in the basis of instantaneous eigenstates,

$$
i \hbar \frac{d}{d t}|\xi(t)\rangle=\left[\varepsilon(t) \sigma_{3}+\hbar \hat{a}(t)\right]|\xi(t)\rangle
$$

with the off-diagonal perturbation $\hat{a}(t)=-U^{\dagger} i \partial_{t} U$. A dominancy balance among the terms gives the condition for the accuracy of the zeroth-order approximation,

$$
\frac{|\varepsilon(t)|}{\hbar} \gg|\hat{a}(t)|
$$

or in scaled time $x=t / \tau$,

$$
\frac{|\varepsilon(x)| \tau}{\hbar} \gg|\hat{a}(x)| .
$$

For our model of the dynamics, $\lambda \propto \tau$ is the largest scale and $|\hat{a}| \sim 1$. The condition Eq. (44) must be maintained at all points on the contour. Applying Eq. (44) on the real axis, where $|\varepsilon(x)| \sim U_{H}$, gives the adiabaticity condition $\lambda \gg 1$. Additionally, in order to connect two Riemann sheets, the contour must pass between two turning points (see Fig. 3), where $|\varepsilon(x)| \sim \delta U_{H}$, giving the condition $\delta \lambda \gg 1$.

Beginning instead from an intuitive approach, we can evaluate the adiabaticity of the dynamics along a given contour. To test whether a given contour is adequate, we can exploit the analogy between quasiadiabatic dynamics and semiclassical scattering. Recall the semiclassical criterion

$$
\frac{\Delta(\Lambda)}{\Lambda} \sim \frac{d \Lambda}{d t} \ll 1
$$

The analog of the de Broglie wavelength $\lambda(x)=2 \pi \hbar / p(x)$ in scattering problems is the period $\Lambda(t) \equiv 2 \pi \hbar / \varepsilon(t)$. In other words, the condition (45) says that the change of period over the course of one period is small. We now require that the semiclassical criterion be obeyed everywhere along an admissible contour, i.e., one that connects the two Riemann sheets. To find an admissible contour, we must appeal to the analytic structure of the eigenenergy $\varepsilon(t)$; see Fig. 3. For clarity we will focus on the time interval $t<0$ and operate under the approximation (28). The singularities of $\varepsilon(t)$ are branch points at $t=-(T / 2)-\tau \ln (-1 \pm i \delta)$ and poles at $t=$ $-(T / 2)-\tau \ln (-1)$. If we agree to define a branch cut connect- 
ing the nearest and next nearest branch points to the real time axis,

$$
\begin{aligned}
& t_{1}=-\frac{T}{2}-\tau \ln \sqrt{1+\delta^{2}}+i \tau[\pi-\arctan (\delta)], \\
& t_{3}=-\frac{T}{2}-\tau \ln \sqrt{1+\delta^{2}}+i \tau[\pi+\arctan (\delta)],
\end{aligned}
$$

respectively, then an admissible contour is one that crosses this branch cut exactly once. For the semiclassical criterion to be obeyed, the admissible contour cannot pass too close to a branch point. In essence, if $\delta$ is too small, the contour is pinched between the branch points $t_{1}$ and $t_{3}$. Evaluating the maximum of $d \Lambda / d t$ on a contour $\mathcal{C}$ that crosses the branch cut between $t=t_{1}$ and $t=t_{3}$ we arrive at the condition $\delta$ $\gg \lambda^{-1}$. Together with the adiabatic limit $\lambda \gg 1$, we have the following conditions on the interdependence of the physical parameters:

$$
\begin{gathered}
\lambda \sim \frac{U_{H} \tau}{\hbar} \gg 1, \\
\delta \lambda \sim \frac{\delta U_{H} \tau}{\hbar} \gg 1 .
\end{gathered}
$$

Each of these dimensionless quantities is a product of a characteristic energy and time scale. If these conditions are not satisfied, there does not exist a contour on which the motion is adiabatic. The integrability (Sec. IV) of our model allows us to investigate the intermediate regime $\lambda \gg 1$ and $\delta \lambda \ll 1$, where Dykhne's formula cannot be justified with the analysis of Hwang and Pechukas.

\section{IDENTIFICATION WITH AN INTEGRABLE MODEL}

The result obtained by Dykhne's formula in Sec. III A is now shown to be equivalent to the exact result for an integrable model in the appropriate limit.

Under the approximations (28) for the time intervals $t$ $<0$ and $t>0$, the Hamiltonian

$$
\mathcal{H}_{\text {eff }}=-\frac{U_{H}}{2}\left(\begin{array}{cc}
1 & t_{H} \\
t_{H} & -1
\end{array}\right)
$$

is approximated by

$$
H_{\mathrm{eff}} \approx\left\{\begin{array}{l}
\mathcal{H}_{<}, \quad t<0 \\
\mathcal{H}_{>}, \quad t>0,
\end{array}\right.
$$

with

$$
\begin{gathered}
H_{<}=-\frac{U_{H}}{2}\left(\begin{array}{cc}
1 & \frac{\delta}{1+e^{-(t / \tau)-(T / 2 \tau)}} \\
\frac{\delta}{1+e^{-(t / \tau)-(T / 2 \tau)}} & -1
\end{array}\right), \\
H_{>}=-\frac{U_{H}}{2}\left(\begin{array}{cc}
1 & \frac{\delta}{1+e^{(t / \tau)-(T / 2 \tau)}} \\
\frac{\delta}{1+e^{(t / \tau)-(T / 2 \tau)}} & -1
\end{array}\right)
\end{gathered}
$$

The Hamiltonians $\mathcal{H}_{<}$and $\mathcal{H}_{>}$can be obtained as a special case of

$$
\mathcal{H}_{\text {exact }}=\left(\begin{array}{cc}
b & a+c \tanh \frac{x}{2} \\
a+c \tanh \frac{x}{2} & -b
\end{array}\right)
$$

by identifying $\pm c=a=-\delta \lambda / 2, b=-\lambda$ and rescaling time $x$ $=t / \tau \pm T / 2 \tau$, respectively. The Schrödinger equation

$$
i \partial_{x}|\psi(x)\rangle=\mathcal{H}_{\text {exact }}|\psi(x)\rangle
$$

is exactly solvable ${ }^{35}$ (see the Appendix).

In analogy with one-dimensional scattering, the transition amplitude from a singly occupied state $\left|S_{1}\right\rangle$ to a doubly occupied state $\left|S_{2}\right\rangle$ may be viewed as an off-diagonal element of the scattering matrix $\mathcal{S}$ that connects the coefficients of the asymptotic final states to the asymptotic initial states. The asymptotic states are the limit as $t \rightarrow \pm \infty$ of the instantaneous eigenstates $\left|\xi_{1,2}(t)\right\rangle$ of $\mathcal{H}_{\text {eff }}$ corresponding to eigenvalues $\mp \varepsilon(t)$, respectively,

$$
\begin{gathered}
\left|\xi_{1}(t)\right\rangle=\frac{1}{\sqrt{2 \varepsilon}}\left(\begin{array}{r}
-\sqrt{\varepsilon-\lambda} \\
\sqrt{\varepsilon+\lambda}
\end{array}\right), \\
\left|\xi_{2}(t)\right\rangle=\frac{1}{\sqrt{2 \varepsilon}}\left(\begin{array}{c}
\sqrt{\varepsilon+\lambda} \\
\sqrt{\varepsilon-\lambda}
\end{array}\right) .
\end{gathered}
$$

The leading behavior of the long-time asymptotics of a general solution has the form

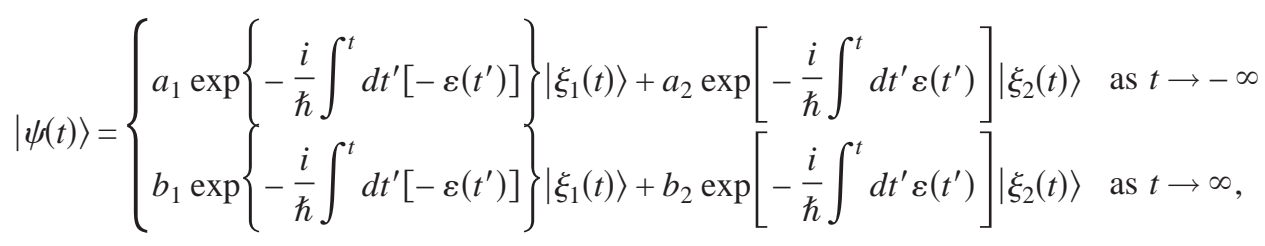


and the scattering matrix relates the coefficients,

$$
\left(\begin{array}{l}
b_{1} \\
b_{2}
\end{array}\right)=\mathcal{S}\left(\begin{array}{l}
a_{1} \\
a_{2}
\end{array}\right) \text {. }
$$

Referring to the statement of our problem in Sec. III, the amplitude of final double occupancy is the element $\mathcal{S}_{21}$ of the scattering matrix, which we parametrize as

$$
\mathcal{S}=\left(\begin{array}{cc}
\mathcal{S}_{11} & \mathcal{S}_{12} \\
-\mathcal{S}_{12}^{*} & \mathcal{S}_{11}^{*}
\end{array}\right) \text {. }
$$

The scattering matrices $\mathcal{S}_{<}$and $\mathcal{S}_{>}$associated with the Hamiltonians $\mathcal{H}_{<}$and $\mathcal{H}_{>}$may be obtained by substitution from the exact scattering matrix $\mathcal{W}$ (derived in the Appendix) associated with the Hamiltonian $\mathcal{H}_{\text {exact }}$. By the symmetry of the pulse, we have $\mathcal{S}_{>}=\mathcal{S}_{<}^{\dagger}$. Patching together the two domains of time evolution (49), we find the scattering matrix

$$
\mathcal{S}=\mathcal{S}_{<}^{\dagger} \exp \left(\frac{i}{\hbar} \sigma_{3} \operatorname{Re} \int_{t_{1}}^{t_{2}} d t \varepsilon(t)\right) \mathcal{S}_{<}
$$

where the integral of the exponent has been estimated in Eq. (36) and the elements of $\mathcal{S}_{<}$are obtained from $\mathcal{W}$,

$$
\left(\mathcal{S}_{<}\right)_{11}=\sqrt{\frac{2 \mu}{\mu+\lambda}} \frac{\Gamma(i 2 \lambda) \Gamma(i 2 \mu)}{\Gamma(i \mu+i \lambda+i \delta \lambda) \Gamma(i \mu+i \lambda-i \delta \lambda)},
$$

$$
\left(\mathcal{S}_{<}\right)_{12}=\sqrt{\frac{2 \mu}{\mu-\lambda}} \frac{\Gamma(i 2 \lambda) \Gamma(-i 2 \mu)}{\Gamma(-i \mu+i \lambda+i \delta \lambda) \Gamma(-i \mu+i \lambda-i \delta \lambda)}
$$

where $\mu=\lambda \sqrt{1+\delta^{2}}$. Dykhne's formula (34) for $P_{<}$is recovered as exactly the leading term of $\left|\left(\mathcal{S}_{<}\right)_{21}\right|^{2}$ in the limit $\lambda, \delta \lambda \gg 1$

$$
\begin{aligned}
P_{<} & =\left|\left(\mathcal{S}_{<}\right)_{21}\right|^{2} \\
& =\frac{\sinh \left[\pi \lambda\left(\sqrt{1+\delta^{2}}-1+\delta\right)\right] \sinh \left[-\pi \lambda\left(\sqrt{1+\delta^{2}}-1-\delta\right)\right]}{\sinh (2 \pi \lambda) \sinh \left(2 \pi \lambda \sqrt{1+\delta^{2}}\right)} \\
& \sim e^{-2 \pi \lambda\left(1+\sqrt{1+\delta^{2}}-\delta\right)}\left(1-e^{-2 \pi \lambda\left(\delta+1-\sqrt{1+\delta^{2}}\right)}+\cdots\right) .
\end{aligned}
$$

The nonperturbative corrections are typically very small. For $\lambda=2$ and $\delta=1 / 2$, the relative contribution of the second term of Eq. (62) is less than 1\%. This accounts for the excellent agreement in Figs. 4-6, between the probability of double occupancy as given by the semiclassical result (35) based on Dykhne's formula and the result of a numerical integration of the Schrödinger equation. We can interpret the subleading term in the parentheses of Eq. (62) as the contribution from the contour $\mathcal{C}$ of Fig. 8, which crosses the branch cut three times. The sign of the correction is negative and arises from the factor $e^{-i \alpha_{1}}$ associated with matching the basis (38) across the branch cut. Similarly, it may be possible to obtain further subdominant corrections to Dykhne's formula by summing over all inequivalent complex paths that give distinct positive values for $\operatorname{Im} \int_{\mathcal{C}} d t \varepsilon(t) .{ }^{36}$ For many physical

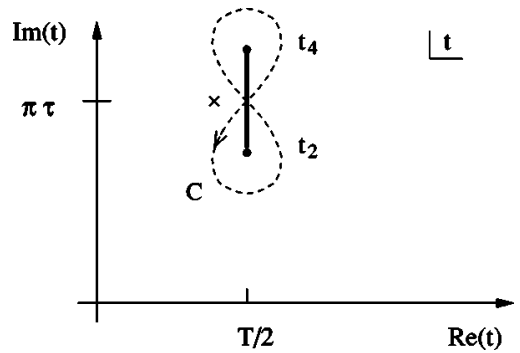

FIG. 8. The contour $\mathcal{C}$ gives a subdominant correction to Dykhne's formula.

problems this type of nonperturbative correction is dominated by perturbative corrections along the contour-those mentioned in Sec. III C. The striking absence of perturbative corrections in the limit $\lambda, \delta \lambda \gg 1$, is a unique artifact of the integrability of our model.

With a knowledge of the exact result, we can also investigate the intermediate regime $\lambda \gg 1$ and $\delta \lambda \ll 1$, where the analysis of Hwang and Pechukas (Sec. III B) cannot be used to prove Dykhne's formula. In this limit, the transition probability (61) becomes

$$
P_{<} \sim(2 \pi \delta \lambda)^{2} e^{-4 \pi \lambda} .
$$

Although Dykhne's formula does not apply in this limit because $\delta^{-1}$ and not $\lambda$ is the largest scale, it nevertheless gives the correct controlling factor $e^{-4 \pi \lambda}$ of Eq. (63), except for $\delta$ that are exponentially small with respect to $\lambda$. This exponential factor is resilient and remains the controlling factor for a range of parameters beyond the naive expectation based on the arguments of Sec. III C.

\section{CONCLUSIONS}

The dynamics of two coupled quantum dot spin qubits can be mapped to an effective two-level system, where nonadiabatic transitions correspond to double occupancy. We have estimated the probability of final double occupancy with Dykhne's formula. In the adiabatic regime, the pervasive feature of transitions is their exponential suppression by a dimensionless adiabaticity parameter $\lambda$. Our main result (35) was expressed in terms of the dimensionless quantities $\lambda, \delta$, and $\eta$. An integral constraint (21) on the swapping operation gives one relation among the three dimensionless parameters. The problem is uniquely defined by specifying any two, and in a solid-state setting, conservative estimates are $\lambda \approx 2$ and $\delta \approx 1 / 2$. The probability of double occupancy $P \approx 10^{-10}$ is sufficiently rare that the operation of a quantum gate will not be obstructed by this type of error. It is noteworthy that the probability of double occupancy (35) has nodes for

$$
k \pi=\operatorname{Re} \int_{t_{1}}^{t_{2}} d t \varepsilon(t) \approx \sqrt{1+\delta^{2}} \lambda \eta, \quad k \in \mathbf{Z} .
$$

However, this property is not immediately relevant to the suppression of transitions, because the oscillatory factor $\sin ^{2}\left(\sqrt{1+\delta^{2}} \lambda \eta\right)$ of Eq. (35) vanishes algebraically and for it 
to provide an improvement upon the exponentially small factor from Dykhne's formula, the argument would have to be tuned exponentially close to $k \pi$. Thus, naively the errors associated with inaccuracies in satisfying the integral swapping constraint (21) will be much greater than double occupancy errors. Other important sources of error are dephasing and decoherence of the qubit states.

We have reviewed a physically motivated derivation of Dykhne's formula. ${ }^{33}$ The theory of semiclassical approximations underlies Dykhne's formula and its validity is appropriately judged within that framework. The semiclassical estimates obtained from this approach are in excellent agreement with numerical simulations of the full quantummechanical time evolution. The corrections to Dykhne's formula are of two types: perturbative and nonperturbative. The former appears to vanish for integrable models, and we have interpreted a nonperturbative correction as the contribution of a contour in the complex time plane. ${ }^{36}$

\section{ACKNOWLEDGMENTS}

A.G.A. and R.R. wish to thank Harold Metcalf for helpful discussions. J.S. and D.L. acknowledge financial support from the Swiss NSF, the NCCR Nanoscience, EU RTN Spintronics, DARPA, ARO, and ONR. A.G.A. received support from the Theory Institute for Strongly Correlated and Complex Systems at BNL as well as from NSF Grant No. DMR0348358.

\section{APPENDIX: INTEGRABLE DYNAMICS}

The following Hamiltonian, ${ }^{35}$ which possesses three independent parameters,

$$
\mathcal{H}_{\text {exact }}(x)=\left(\begin{array}{cc}
b & a+c \tanh \frac{x}{2} \\
a+c \tanh \frac{x}{2} & -b
\end{array}\right)
$$

has integrable dynamics in the sense that the Schrödinger equation

$$
\left[i \partial_{x}-\mathcal{H}_{\text {exact }}(x)\right]|\psi(x)\rangle=0 \text { with }|\psi(x)\rangle=\left(\begin{array}{l}
c_{1}(x) \\
c_{2}(x)
\end{array}\right)
$$

has a solution in terms of special functions. Our aim is to construct the scattering matrix $\mathcal{W}$ associated with the dynamical problem. For clarity of presentation, we will consider the Hamiltonian

$$
\mathcal{H}^{\prime}(x)=\left(\begin{array}{cc}
a+c \tanh \frac{x}{2} & b \\
b & -\left(a+c \tanh \frac{x}{2}\right)
\end{array}\right),
$$

which differs from $\mathcal{H}_{\text {exact }}$ by a constant unitary transformation $V$,

$$
V^{\dagger} \mathcal{H}_{\text {exact }} V=\mathcal{H}^{\prime} \text { with } V=\frac{1}{\sqrt{2}}\left(\begin{array}{cc}
1 & 1 \\
1 & -1
\end{array}\right) .
$$

Introducing the mixing angle $\phi$ defined as

$$
\tan \phi=\frac{b}{a+c \tanh \frac{x}{2}},
$$

the instantaneous eigenstates $\left|\chi_{1,2}(x)\right\rangle$ corresponding to instantaneous eigenvalues

$$
\pm \varepsilon(x)= \pm \sqrt{b^{2}+\left(a+c \tanh \frac{x}{2}\right)^{2}},
$$

respectively, are parametrized as

$$
\left|\chi_{1}(t)\right\rangle=\frac{1}{\sqrt{2 \varepsilon}}\left(\begin{array}{l}
\sqrt{\varepsilon+\left(a+c \tanh \frac{x}{2}\right)} \\
\sqrt{\varepsilon-\left(a+c \tanh \frac{x}{2}\right)}
\end{array}\right)=\left(\begin{array}{r}
\cos \frac{\phi}{2} \\
\sin \frac{\phi}{2}
\end{array}\right),
$$

$$
\left|\chi_{2}(t)\right\rangle=\frac{1}{\sqrt{2 \varepsilon}}\left(\begin{array}{c}
-\sqrt{\varepsilon-\left(a+c \tanh \frac{x}{2}\right)} \\
\sqrt{\varepsilon+\left(a+c \tanh \frac{x}{2}\right)}
\end{array}\right)=\left(\begin{array}{r}
-\sin \frac{\phi}{2} \\
\cos \frac{\phi}{2}
\end{array}\right) \text {. }
$$

The two-state Schrödinger equation may be converted to a second-order differential equation for the components $c_{1,2}$ $=c_{1,2}(x)$,

$$
\partial_{x}^{2} c_{1,2}+Q_{1,2}(x) c_{1,2}=0
$$

where

$$
Q_{1,2}(x)=b^{2}+\left(a+c \tanh \frac{x}{2}\right)^{2} \pm i \frac{1}{2} c \operatorname{sech}^{2} \frac{x}{2} .
$$

Changing the dependent and independent variables as $c_{1,2}(x)=z^{ \pm i \nu}(z-1)^{i \mu} w_{1,2}(z)$ and $z=\frac{1}{2}[1+\tanh (x / 2)]$, transforms Eq. (A9) into the standard form of the Gauss hypergeometric equation, for example, for $w_{1}(x)$

$$
z(1-z) \partial_{z}^{2} w_{1}+[k-z(i+j-1)] \partial_{z} w_{1}-i j w_{1}=0,
$$

where the arguments and exponents are defined as follows:

$$
\begin{gathered}
i=i \mu+i \nu-i 2 c, \\
j=i \mu+i \nu+i 2 c+1, \\
k=i 2 \nu+1, \\
\nu=\varepsilon(-\infty)=\sqrt{b^{2}+(a-c)^{2}}, \\
\mu=\varepsilon(\infty)=\sqrt{b^{2}+(a+c)^{2}} .
\end{gathered}
$$

The two linearly independent solutions $u_{1}(z)$ and $v_{1}(z)$ of the differential equation for $w_{1}(z)$ are 


$$
\begin{gathered}
u_{1}(i, j, k ; z)=z^{i \nu}(z-1)_{2}^{i \mu} F_{1}(i, j, k ; z), \\
\begin{aligned}
v_{1}(l, m, n ; z) & =z^{1-k} u_{1}(i-k+1, j-k+1,2-k ; z) \\
& =z^{-i \nu}(z-1)^{i \mu}{ }_{2} F_{1}(i-k+1, j-k+1,2-k ; z),
\end{aligned}
\end{gathered}
$$

where ${ }_{2} F_{1}(i, j, k ; z)$ is the Gauss hypergeometric function. ${ }^{37}$

The amplitude of transition may be viewed as the offdiagonal element of the scattering matrix $\mathcal{W}$ that connects the asymptotic final and initial states for $t \rightarrow \pm \infty$ [see Eq. (56)]. The scattering matrix is parametrized as

$$
\mathcal{W}=\left(\begin{array}{cc}
\mathcal{W}_{11} & \mathcal{W}_{12} \\
-\mathcal{W}_{12}^{*} & \mathcal{W}_{11}^{*}
\end{array}\right)
$$

because it has the properties $\mathcal{W}^{\dagger} \mathcal{W}=1$ and $\operatorname{det}(\mathcal{W})=1$. From the asymptotics of $u_{1}, u_{2}, v_{1}$, and $v_{2}$ in the limits $z \rightarrow 0$ and $z \rightarrow 1$, we find

$$
\begin{aligned}
\mathcal{W}_{11}= & \sqrt{\frac{b^{2}+[\mu-(a+c)]^{2}}{b^{2}+[\nu-(a-c)]^{2}}} \\
& \times \frac{\Gamma(1-i 2 \nu) \Gamma(-i 2 \mu)}{\Gamma[1-i(\mu+\nu-2 c)] \Gamma[-i(\mu+\nu+2 c)]},
\end{aligned}
$$

$$
\begin{aligned}
\mathcal{W}_{12}= & \sqrt{\frac{b^{2}+[\mu+(a+c)]^{2}}{b^{2}+[\nu-(a-c)]^{2}}} \\
& \times \frac{\Gamma(1-i 2 \nu) \Gamma(i 2 \mu)}{\Gamma[i(\mu-\nu-2 c)] \Gamma[1+i(\mu-\nu+2 c)]}
\end{aligned}
$$

and

$$
\left|\mathcal{W}_{11}\right|^{2}=\frac{\sinh \pi(2 c+\mu+\nu) \sinh \pi(-2 c+\mu+\nu)}{\sinh 2 \pi \mu \sinh 2 \pi \nu},
$$

$$
\left|\mathcal{W}_{12}\right|^{2}=\frac{\sinh \pi(2 c-\mu+\nu) \sinh \pi(2 c+\mu-\nu)}{\sinh 2 \pi \mu \sinh 2 \pi \nu} .
$$

*Electronic address: rrequist@grad.physics.sunysb.edu

†Electronic address: John.Schliemann@unibas.ch

¥Electronic address: Alexandre.Abanov@ stonybrook.edu

§Electronic address: Daniel.Loss@unibas.ch

${ }^{1}$ For a general overview, see, e.g., M. A. Nielsen and I. L. Chuang, Quantum Computation and Quantum Information (Cambridge University Press, Cambridge, 2000).

${ }^{2}$ D. Loss and D. P. DiVincenzo, Phys. Rev. A 57, 120 (1998).

${ }^{3}$ V. Privman, I. D. Vagner, and G. Kventsel, Phys. Lett. A 239, 141 (1998).

${ }^{4}$ B. E. Kane, Nature (London) 393, 133 (1998).

${ }^{5}$ C. H. W. Barnes, J. M. Shilton, and A. M. Robinson, Phys. Rev. B 62, 8410 (2000).

${ }^{6}$ J. Levy, Phys. Rev. A 64, 052306 (2001).

${ }^{7}$ T. D. Ladd, J. R. Goldman, F. Yamaguchi, Y. Yamamoto, E. Abe, and K. M. Itoh, Phys. Rev. Lett. 89, 017901 (2002).

${ }^{8}$ G. Burkard, D. Loss, and D. P. DiVincenzo, Phys. Rev. B 59, 2070 (1999).

${ }^{9}$ X. Hu and S. Das Sarma, Phys. Rev. A 61, 062301 (2000); 64, 042312 (2001).

${ }^{10}$ J. Schliemann, D. Loss, and A. H. MacDonald, Phys. Rev. B 63, 085311 (2001).

${ }^{11}$ M. Friesen, P. Rugheimer, D. E. Savage, M. G. Lagally, D. W. van der Weide, R. Joynt, and M. A. Eriksson, Phys. Rev. B 67, 121301 (2003).

${ }^{12}$ L. M. K. Vandersypen, R. Hanson, L. H. Willems van Beveren, J. M. Elzerman, J. S. Greidanus, S. De Franceschi, and L. P. Kouwenhoven, Phys. Rev. B 67, 161308 (2003).

${ }^{13}$ R. Hanson, B. Witkamp, L. M. K. Vandersypen, L. H. Willems van Beveren, J. M. Elzerman, and L. P. Kouwenhoven, Phys.
Rev. Lett. 91, 196802 (2003).

${ }^{14}$ D. Stepanenko, N. E. Bonesteel, D. P. DiVincenzo, G. Burkard, and D. Loss, Phys. Rev. B 68, 115306 (2003).

${ }^{15}$ For a review, see G. Burkard and D. Loss in Semiconductor Spintronics and Quantum Computation, edited by D. D. Awschalom, D. Loss, and N. Samarth (Springer, Berlin, 2002).

${ }^{16}$ J. Schliemann, J. I. Cirac, M. Kus, M. Lewenstein, and D. Loss, Phys. Rev. A 64, 022303 (2001).

${ }^{17}$ K. Eckert, J. Schliemann, D. Bruss, and M. Lewenstein, Ann. Phys. (N.Y.) 299, 88 (2002).

${ }^{18}$ L. D. Landau, Phys. Z. Sowjetunion 1, 88 (1932); 2, 46 (1932).

${ }^{19}$ C. Zener, Proc. R. Soc. London, Ser. A 137, 696 (1932).

${ }^{20}$ E. C. G. Stueckelberg, Helv. Phys. Acta 5, 369 (1932).

${ }^{21}$ N. Rosen and C. Zener, Phys. Rev. 40, 502 (1932).

${ }^{22}$ S. Stenholm, in Quantum Dynamics of Simple Systems, Proceedings of the 44th Scottish Universities Summer School of Physics, Stirling, 1994, edited by G.-L. Oppo, S. M. Barnett, E. Riis, and M. Wilkinson (Scottish Universities Summer School in Physics, Bristol, 1996), p. 267.

${ }^{23}$ A. M. Dykhne, Sov. Phys. JETP 14, 941 (1962).

${ }^{24}$ D. P. DiVincenzo, Phys. Rev. A 51, 1015 (1995).

${ }^{25}$ G. Burkard, G. Seelig, and D. Loss, Phys. Rev. B 62, 2581 (2000).

${ }^{26}$ M. V. Berry, Proc. R. Soc. London, Ser. A 430, 405 (1990).

${ }^{27}$ D. Loss, D. P. DiVincenzo, and G. Grinstein, Phys. Rev. Lett. 69, 3232 (1992).

${ }^{28}$ M. N. Leuenberger and D. Loss, Phys. Rev. B 63, 054414 (2001).

${ }^{29}$ A. Bambini and P. R. Berman, Phys. Rev. A 23, 2496 (1981).

${ }^{30}$ E. J. Robinson, Phys. Rev. A 29, 1665 (1984).

${ }^{31}$ N. V. Vitanov, J. Phys. B 27, 1351 (1994). 
${ }^{32}$ E. J. Robinson, J. Phys. B 28, L169 (1995).

${ }^{33}$ J-T. Hwang and P. Pechukas, J. Chem. Phys. 67, 4640 (1977).

${ }^{34}$ A. Shapere and F. Wilczek, Geometric Phases in Physics (World Scientific, Singapore, 1989).

${ }^{35}$ N. B. Naronzhny and A. I. Nikishov, Sov. J. Nucl. Phys. 11, 596
(1970).

${ }^{36}$ K.-S. Lam, J. Phys. A 29, 1055 (1996).

${ }^{37}$ I. S. Gradshteyn and I. M. Ryzhik, Table of Integrals, Series, and Products, 6th ed. (Academic, San Diego, CA, 2000). 